\title{
TWO TYPES OF EXTERNAL LINEAR MODELS USED FOR ADAPTIVE CONTROL OF CONTINUOUS STIRRED TANK REACTOR
}

\author{
Jiri Vojtesek and Petr Dostal \\ Faculty of Applied Informatics \\ Tomas Bata University in Zlin \\ Nad Stranemi 4511, 76005 Zlin, Czech Republic \\ E-mail: \{vojtesek,dostalp\}@fai.utb.cz
}

\section{KEYWORDS}

Adaptive control, Polynomial approach, Pole-placement method, Recursive identification, CSTR

\begin{abstract}
The main goal of this contribution is to show simulation results for two types of External Linear Models (ELM) used in the adaptive control as a linear representation of the originally nonlinear system. The nonlinearity is dispathed with the use of recursive identification which recomputes parameters of ELM in each step according to actual state of the system. The controller design employes polynomial approach with pole-placement method and spectral factorization and all these techniques together satisfies basic control requirements. The verification was done on the mathematical model of Continuous Stirred Tank Reactor (CSTR) as a typical member of the nonlinear equipment widely used in chemical industry.
\end{abstract}

\section{INTRODUCTION}

The adaptive control ( $\AA$ ström and Wittenmark 1989) is not new control technique but his advantages could be found in the big theoretical background and usability to cooperate with other control approaches such as a robust control, a predictive control etc.

Several methods used in adaptive control are introduced for example in (Bobal et al. 2005). The polynomial approach (Kucera 1993) in the control synthesis can be used for systems with negative properties from the control point of view such as nonlinear systems, nonminimum phase systems or systems with time delays. Moreover, the pole-placed method with spectral factorization satisfies basic control requirements such as disturbance attenuation, stability and reference signal tracking.

Although the polynomial synthesis is considered for the continuous-time ELM, the recursive identification with exponential forgetting (Rao and Unbehauen 2005) runs in discrete time which is better from computation and programming point of view. This disagreement could be overcome with the use so called Delta $(\delta$-) models (Middleton and Goodwin 2004) that belong the discrete-time models but parameters approaches to the continuous ones for the small sampling period (Stericker and Sinha 1993).
The nonlinear plant in the verification part is represented by Continuous Stirred Tank Reactor with van der Vusse reaction inside and cooling in the jacket (Chen et al. 1995). The mathematical model of this reactor is described by the set of four nonlinear Ordinary Differential Equations (ODEs) which can be easily solved with standard methods for numerical solving.

All simulation studies were done on mathematical simulation software Matlab, version 6.5.1.

\section{ADAPTIVE CONTROL}

The Adaptive control is based on the quality of real organisms which can change behavior according to environmental conditions. This process is usually called "adaptation". There are several ways of use of the adaptation.

The adaptive approach in this work is based on choosing an external linear model (ELM) of the original nonlinear system whose parameters are recursively identified during the control. The choice of ELM is usually based on the dynamic analysis of the system. The possible change of the ELM parameters is taken into account by the recursive identification of ELM during the control.

\section{External Linear Model (ELM)}

There are several types of ELM such as continuous-time and discrete-time models. ELM used here is described generally by the transfer function (TF):

$$
G(s)=\frac{Y(s)}{U(s)}=\frac{b(s)}{a(s)}
$$

As it can be seen, this model belongs to the class of continuous-time (CT) models. The identification of such processes is not very easy.

One way, how we can overcome this problem is the use of so called $\delta$-model. This model belongs to the class of discrete models but its parameters are close to the continuous ones for very small sampling period as it proofed in (Stericker and Sinha 1993).

The $\delta$-model introduces a new complex variable $\gamma$ computed as (see (Mukhopadhyay et al. 1992)):

$$
\gamma=\frac{z-1}{\beta \cdot T_{v} \cdot z+(1-\beta) \cdot T_{v}}
$$


Where $\beta$ is an optional parameter from the interval $0 \leq \beta \leq 1$ and $T_{v}$ denotes a sampling period. It is clear that we can obtain infinite number of $\delta$-models for various $\beta$. A so called forward $\delta$-model for $\beta=0$ was used and $\gamma$ operator is then

$$
\gamma=\frac{z-1}{T_{v}}
$$

The continuous model (1) is then rewritten to the form

$$
a^{\delta}(\delta) y\left(t^{\prime}\right)=b^{\delta}(\delta) u\left(t^{\prime}\right)
$$

where polynomials $a^{\delta}(\delta)$ and $b^{\delta}(\delta)$ are discrete polynomials and their coefficients are different from those of the CT model $a(s)$ and $b(s)$. Time $t^{\prime}$ is discrete time.

Now we can introduce substitution $t^{\prime}=k-n$ for $k \geq n$ and Equation (4) then will be

$$
\begin{aligned}
& \delta^{n} y(k-n)=b_{m}^{\delta} \delta^{m} u(k-n)+\ldots+b_{1}^{\delta} \delta u(k-n)+b_{0}^{\delta} u(k-n)- \\
& -a_{n-1}^{\delta} \delta^{n-1} y(k-n)-\ldots-a_{1}^{\delta} \delta y(k-n)-a_{0}^{\delta} y(k-n)
\end{aligned}
$$

where individual elements are

$$
\begin{aligned}
& \delta^{i} y(k-n)=\sum_{j=0}^{i} \frac{(-1)^{j}}{T_{v}^{i}}\left(\begin{array}{l}
i \\
j
\end{array}\right) y(k-n+i-j), \text { for } i=0,1, \ldots, n(6) \\
& \delta^{l} u(k-n)=\sum_{j=0}^{l} \frac{(-1)^{j}}{T_{v}^{l}}\left(\begin{array}{l}
l \\
j
\end{array}\right) u(k-n+l-j), \text { for } l=0,1, \ldots, m(7)
\end{aligned}
$$

And the individual parts in Equation (5) can be written as

$$
\begin{array}{cl}
y_{\delta}(k)=\delta^{n} y(k-n), & u_{\delta}(k-n+m)=\delta^{m} u(k-n), \\
y_{\delta}(k-1)=\delta^{n-1} y(k-n), & u_{\delta}(k-n+m-1)=\delta^{m-1} u(k-n), \\
\vdots & \vdots \\
y_{\delta}(k-n+1)=\delta y(k-n) & u_{\delta}(k-n+1)=\delta u(k-n), \\
y_{\delta}(k-n)=y(k-n) & u_{\delta}(k-n)=u(k-n)
\end{array}
$$

The regression vector $\varphi_{\delta}$ is then

$$
\begin{aligned}
& \varphi_{\delta}(k-1)=\left[-y_{\delta}(k-1), \ldots,-y_{\delta}(k-n+1),-y_{\delta}(k-n),\right. \\
& \left.u_{\delta}(k+m-n), u_{\delta}(k+m-1-n), \ldots, u_{\delta}(k-n+1), u_{\delta}(k-n)\right]^{T}
\end{aligned}
$$

and the vector of parameters $\boldsymbol{\theta}_{\delta}$ is generally

$$
\boldsymbol{\theta}_{\delta}(k)=\left[a_{n-1}^{\delta}, \ldots, a_{1}^{\delta}, a_{0}^{\delta}, b_{m}^{\delta}, b_{m-1}^{\delta}, \ldots, b_{1}^{\delta}, b_{0}^{\delta}\right]^{T}
$$

which is computed from the differential equation

$$
y_{\delta}(k)=\theta_{\delta}^{T}(k) \cdot \varphi_{\delta}(k-1)+e(k)
$$

where $e(k)$ is a general random immeasurable component.

\section{Identification of ELM parameters}

The Recursive Least-Squares (RLS) method is used for the parameter estimation in this work. The RLS method is well-known and widely used for the parameter estimation. It is usually modified with some kind of forgetting, exponential or directional. Parameters of the identified system can vary during the control which is typical for nonlinear systems and the use of some forgetting factor could result in better output response. The basic RLS method is described by the set of equations:

$$
\begin{aligned}
& \varepsilon(k)=y(k)-\boldsymbol{\varphi}^{T}(k) \cdot \hat{\boldsymbol{\theta}}(k-1) \\
& \gamma(k)=\left[1+\boldsymbol{\varphi}^{T}(k) \cdot \mathbf{P}(k-1) \cdot \boldsymbol{\varphi}(k)\right]^{-1} \\
& \boldsymbol{L}(k)=\gamma(k) \cdot \mathbf{P}(k-1) \cdot \boldsymbol{\varphi}(k) \\
& \mathbf{P}(k)=\frac{1}{\lambda_{1}(k-1)}\left[\mathbf{P}(k-1)-\frac{\mathbf{P}(k-1) \cdot \boldsymbol{\varphi}(k) \cdot \boldsymbol{\varphi}^{T}(k) \cdot \mathbf{P}(k-1)}{\lambda_{1}(k-1)+\boldsymbol{\varphi}^{T}(k) \cdot \mathbf{P}(k-1) \cdot \boldsymbol{\varphi}(k)}\right] \\
& \hat{\boldsymbol{\theta}}(k)=\hat{\boldsymbol{\theta}}(k-1)+\boldsymbol{L}(k) \varepsilon(k)
\end{aligned}
$$

RLS with the changing exp. forgetting is used for parameter estimation, where the changing forgetting factor $\lambda_{1}$ is computed from the equation

$$
\lambda_{1}(k)=1-K \cdot \gamma(k) \cdot \varepsilon^{2}(k)
$$

Where $K$ is small number, in our case $K=0.001$.

\section{Control System Synthesis}

The simple control system configuration with one degree-of-freedom (1DOF) was used here. As you can see in Figure 1, this basic configuration has controller in the feedback part.

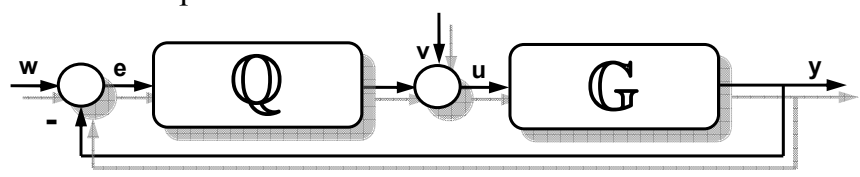

Figure 1: 1DOF control configuration

The block $G$ denotes transfer function (1) of controlled plant, $w$ is the reference signal (wanted value), $v$ is disturbance, $e$ is used for control error, $u$ is control variable and $y$ is a controlled output. The transfer function of the feedforward part $Q(s)$ of the controller is designed with the use of polynomial synthesis:

$$
\tilde{Q}(s)=\frac{q(s)}{s \cdot \tilde{p}(s)}
$$

where degrees of polynomials $\tilde{p}(s)$ and $q(s)$ are computed from:

$$
\begin{aligned}
& \operatorname{deg} q(s)=\operatorname{deg} a(s)+\operatorname{deg} f(s)-1 \\
& \operatorname{deg} \tilde{p}(s) \geq \operatorname{deg} a(s)-1
\end{aligned}
$$

and parameters of these polynomials are computed by the Method of uncertain coefficients which compares coefficients of individual $s$-powers from the Diophantine equation, e.g. (Kucera 1993):

$$
a(s) \cdot s \cdot \tilde{p}(s)+b(s) \cdot q(s)=d(s)
$$

The polynomial $d(s)$ on the right side of (16) is an optional stable polynomial. It is obvious, that the degree of this polynomial is:

$$
\operatorname{deg} d(s)=\operatorname{deg} a(s)+\operatorname{deg} \tilde{p}(s)+1
$$

and roots of this polynomial are called poles of the closed-loop and their position affects quality of the control.

This polynomial could be designed for example with the use of Pole-placement method. A choice of roots needs some a priory information about the system's behavior. It is good to connect poles with the parameters of the 
system via spectral factorization. The polynomial $d(s)$ can be then rewritten to the form

$$
d(s)=n(s) \cdot(s+\alpha)^{\operatorname{deg} d-\operatorname{deg} n}
$$

where $\alpha>0$ is an optional coefficient reflecting closedloop poles and stable polynomial $n(s)$ is obtained from the spectral factorization of the polynomial $a(s)$

$$
n^{*}(s) \cdot n(s)=a^{*}(s) \cdot a(s)
$$

The Diophantine equation (16), as it is, is valid for step changes of the reference and disturbance signals which means that $\operatorname{deg} f(s)=1$ in (15). The feedback controller $Q(s)$ ensures stability, load disturbance attenuation and asymptotic tracking of the reference signal. The control system synthesis is done here in continuous time, but recursive identification uses discrete time steps. The resulted, so called "hybrid", controller works in the continuous time but parameters of the polynomials $a(s)$ and $b(s)$ are identified recursively in the sampling period $T_{v}$. This assumption results in the condition, that the parameters of the $\delta$-model are close the continuous ones for the small sampling period.

\section{MODEL OF THE REACTOR}

The controlled process here is represented by the continuous stirred tank reactor (CSTR) with so called van der Vusse reaction inside the reactor (Chen et al. 1995):

$$
\begin{aligned}
& A \stackrel{k_{1}}{\longrightarrow} B \stackrel{k_{2}}{\longrightarrow} C \\
& 2 A \stackrel{k_{3}}{\longrightarrow} D
\end{aligned}
$$

The mathematical model of this reactor is described by the following set of ordinary differential equations (ODE):

$$
\begin{aligned}
\frac{d c_{A}}{d t} & =\frac{q_{r}}{V_{r}}\left(c_{A 0}-c_{A}\right)-k_{1} c_{A}-k_{3} c_{A}{ }^{2} \\
\frac{d c_{B}}{d t} & =-\frac{q_{r}}{V_{r}} c_{B}+k_{1} c_{A}-k_{2} c_{B} \\
\frac{d T_{r}}{d t} & =\frac{q_{r}}{V_{r}}\left(T_{r 0}-T_{r}\right)-\frac{h_{r}}{\rho_{r} c_{p r}}+\frac{A_{r} U}{V_{r} \rho_{r} c_{p r}}\left(T_{c}-T_{r}\right) \\
\frac{d T_{c}}{d t} & =\frac{1}{m_{c} c_{p c}}\left(Q_{c}+A_{r} U\left(T_{r}-T_{c}\right)\right)
\end{aligned}
$$

Table 1: Fixed parameters of the reactor

\begin{tabular}{|cc|}
\hline$k_{01}=2.145 \cdot 10^{10} \mathrm{~min}^{-1}$ & $E_{1} / R=9758.3 \mathrm{~K}$ \\
$k_{02}=2.145 \cdot 10^{10} \mathrm{~min}^{-1}$ & $E_{2} / R=9758.3 \mathrm{~K}$ \\
$k_{03}=1.5072 \cdot 10^{8} \mathrm{~min}^{-1} \cdot \mathrm{mol}^{-1}$ & $E_{3} / R=8560 \mathrm{~K}$ \\
$h_{1}=-4200 \mathrm{~kJ} . \mathrm{kmol}^{-1}$ & $h_{2}=11000 \mathrm{~kJ} . \mathrm{kmol}^{-1}$ \\
$h_{3}=41850 \mathrm{~kJ} . \mathrm{kmol}^{-1}$ & $U=67.2 \mathrm{~kJ} . \mathrm{min}^{-1} \mathrm{~m}^{-2} \mathrm{~K}^{-1}$ \\
$V_{r}=0.01 \mathrm{~m}^{3}$ & $A_{r}=0.215 \mathrm{~m}^{2}$ \\
$c_{p r}=3.01 \mathrm{~kJ} . \mathrm{kg}^{-1} . \mathrm{K}^{-1}$ & $c_{p c}=2.0 \mathrm{~kJ} . \mathrm{kg}^{-1} \cdot \mathrm{K}^{-1}$ \\
$m_{c}=5 \mathrm{~kg}$ & $T_{r 0}=387.05 \mathrm{~K}$ \\
$c_{A 0}=5.1 \mathrm{kmol} . \mathrm{m}^{-3}$ & $c_{B 0}=0 \mathrm{kmol} . \mathrm{m}^{-3}$ \\
$\rho_{r}=934.2 \mathrm{~kg} . \mathrm{m}^{-3}$ & \\
\hline
\end{tabular}

The graphical scheme of this reactor can be seen in Figure 2.

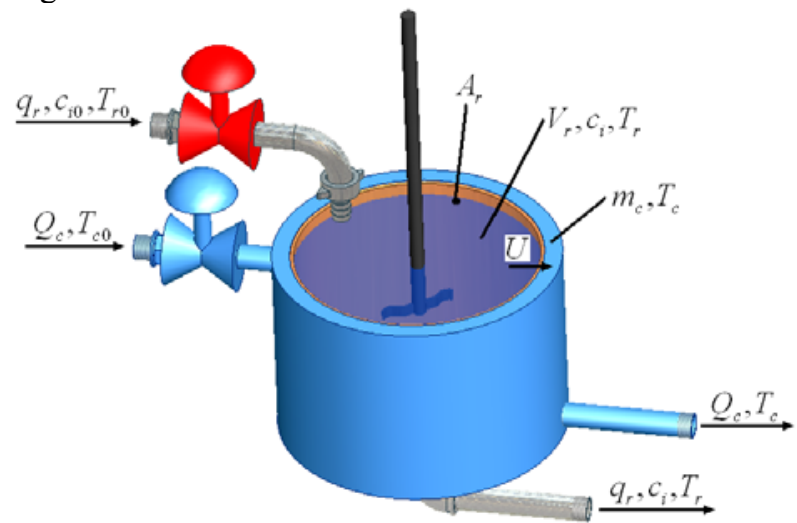

Figure 2: Continuous Stirred Tank Reactor (CSTR)

This set of ODE together with simplifications then mathematically represents examined CSTR reactor. The model of the reactor belongs to the class of lumpedparameter nonlinear systems. Fixed parameters of the system are shown in Table 1.

The reaction heat $\left(h_{r}\right)$ in eq. (21) is expressed as:

$$
h_{r}=h_{1} \cdot k_{1} \cdot c_{A}+h_{2} \cdot k_{2} \cdot c_{B}+h_{3} \cdot k_{3} \cdot c_{A}^{2}
$$

where $h_{i}$ stands for reaction enthalpies.

Nonlinearity can be found in reaction rates $\left(k_{j}\right)$ which are described via Arrhenius law:

$$
k_{j}\left(T_{r}\right)=k_{0 j} \cdot \exp \left(\frac{-E_{j}}{R T_{r}}\right), \text { for } j=1,2,3
$$

where $k_{0}$ represent pre-exponential factors and $E$ are activation energies.

\section{STEADY-STATE AND DYNAMIC ANALYSES}

Steady-state and dynamic analyses can help us with the description of the system's behaviour. Both analyses are discussed for example in (Vojtesek et al. 2008). An optimal working point coming from steady-state analysis is represented by the volumetric flow rate of the reactant $q_{r}=2.4 \cdot 10-3 \mathrm{~m}^{3} \cdot \mathrm{min}^{-1}$ and heat removal of the coolant $Q_{c}=-18.56 \mathrm{~kJ}_{\mathrm{min}} \mathrm{m}^{-1}$. The steady-state values of the state variables for this working point are then

$$
\begin{array}{llrl}
c_{A}^{s} & =2.14 \mathrm{kmol} . \mathrm{m}^{-3} & & c_{B}^{s}=1.09 \mathrm{kmol} . \mathrm{m}^{-3} \\
T_{r}^{s}=387 \mathrm{~K} & T_{c}^{s}=386 \mathrm{~K}
\end{array}
$$

Figure 3 shows course of the output temperature of the reactant, $T_{r}$, after the step change of the input heat removal, $Q_{c}$ in dynamic analysis. These variables are used as an input and an output variables $u(t)$ and $y(t)$ in the adaptive control and

$$
u(t)=\frac{Q_{c}(t)-Q_{c}^{s}}{Q_{c}^{s}} \cdot 100[\%], \quad y(t)=T(t)-T^{s}[K]
$$

What is interesting and can be clearly seen from previous figure, is that the ELM used in adaptive approach described above can be chosen as a transfer function (1) of different orders, e.g. 


$$
G_{1}(s)=\frac{b_{0}}{a_{1} s+a_{0}} ; G_{2}(s)=\frac{b_{1} s+b_{0}}{a_{2} s^{2}+a_{1} s+a_{0}}
$$

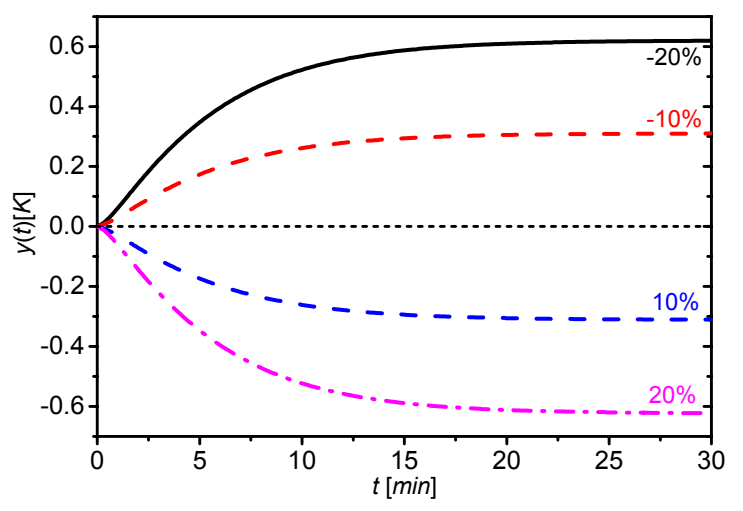

Figure 3: Dynamic analysis for various step changes of the input heat removal $\Delta Q_{c}$

The usability of both TF $G_{1}$ and $G_{2}$ in (26) can be easily tested if we use recursive identification with exponential forgetting described in previous chapters to the dynamic study. Results for the step change of the heat removal $\Delta Q_{c}=+20 \%$ displayed in Figure 4 show that both ELM $G_{1}$ and $G_{2}$ describes output $y(t)$ in proper way and also the recursive identification has no problem with the on-line identification of the simulated data.

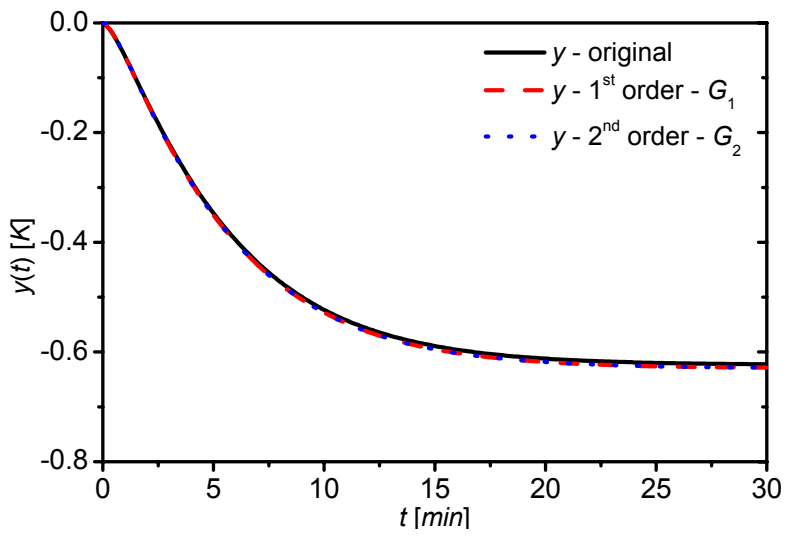

Figure 4: Comparison of the identification with the $1^{\text {st }}$ order and $2^{\text {nd }}$ order TF ELM for $\Delta Q_{c}=+20 \%$

\section{ADAPTIVE CONTROL}

The goal of this chapter is to show the use of different ELM in the way of the order of the transfer fuction. Two ELM transfer functions $G_{1}$ and $G_{2}$ with the first order and the second order with relative order one as displayed in (26) were used.

The presence of the parameters $a_{1}$ and $a_{2}$ in the $G_{1}(s)$ and $G_{2}(s)$ makes these TF multivalent. This feature could be overcome with the condition $a_{1}=1$ for $G_{1}$ and $a_{2}=1$ for $G_{2}$, i.e.

$$
G_{1}(s)=\frac{b_{0}}{s+a_{0}} ; \quad G_{2}(s)=\frac{b_{1} s+b_{0}}{s^{2}+a_{1} s+a_{0}}
$$

As it is written in the theoretical part, the degree of the polynomials $a(s)$ and $b(s)$ in (27) affects the degree of the controller's polynomials $\tilde{p}(s)$ and $q(s)$ in (15) and the degree of the stable optional polynomial $d(s)$ on the right side of the Diophantine equation (16) which is computed from (17). The form of these polynomials and the form of the vector of parameters $\hat{\boldsymbol{\theta}}_{\delta}^{\boldsymbol{T}}$ for both types of ELM are shown in following Table 2.

Table 2: Different polynomials and vectors for both ELM

\begin{tabular}{|c|c|}
\hline$G_{1}(s)=\frac{b_{0}}{s+a_{0}}$ & $G_{2}(s)=\frac{b_{1} s+b_{0}}{s^{2}+a_{1} s+a_{0}}$ \\
\hline$\tilde{Q}(s)=\frac{q_{1} s+q_{0}}{s \cdot p_{0}}$ & $\tilde{Q}(s)=\frac{q_{2} s^{2}+q_{1} s+q_{0}}{s \cdot\left(s+p_{0}\right)}$ \\
$d(s)=\left(s+n_{0}\right) \cdot(s+\alpha)$ & $d(s)=\left(s^{2}+n_{1} s+n_{0}\right) \cdot(s+\alpha)^{2}$ \\
$\hat{\boldsymbol{\theta}}_{\delta}^{\boldsymbol{T}}(k)=\left[a_{0}^{\delta}, b_{0}^{\delta}\right]$ & $\hat{\boldsymbol{\theta}}_{\delta}^{\boldsymbol{T}}(k)=\left[a_{1}^{\delta}, a_{0}^{\delta}, b_{1}^{\delta}, b_{0}^{\delta}\right]$ \\
\hline
\end{tabular}

\section{SIMULATION RESULTS OF CONTROL}

The proposed controller could be tuned via the choice of the parameter $\alpha$ in the polynomial $d(s)$ and this was tested in the following simulation studies. All simulations have the same properties, such as sampling period $T_{v}=0.3 \mathrm{~min}$, simulation time $350 \mathrm{~min}$ and seven different step changes were done during this time. The input variable was limited to $u(t)=<-75 ; 75>\%$ due to the physical properties of the reactor.

The first simulation study for the first order $\operatorname{ELM~} G_{1}(s)$ was done for the position of the root $\alpha=0.04,0.1$ and 0.3 and results are shown in Figure 5 and Figure 6.

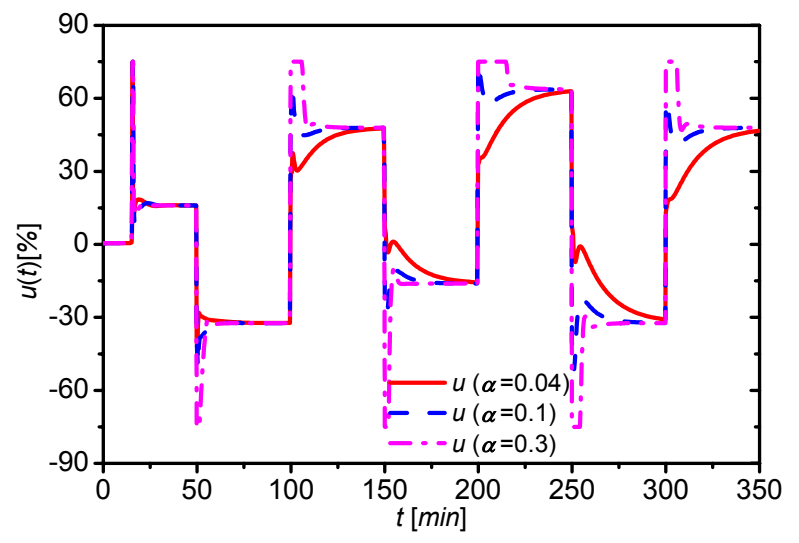

Figure 5: The course of the input variable, $u(t)$, for different value of the parameter $\alpha, 1^{\text {st }}$ order ELM $G_{1}$

It can be clearly seen, that the tuning parameter $\alpha$ results in slower but smoothe output response. Small value makes very quick output response but overshoots from the reference signal. Ideal value should be chosen somewhere between 0.04 and 0.1 . The recursive identification has very good results two although the 
controller does not know about the system behaviour at the begging which is represented by the starting vector of parameters $\hat{\theta}^{T}=[0.1,0.1]$. The only improvement is the use of proportional controller for initial 15 minutes.

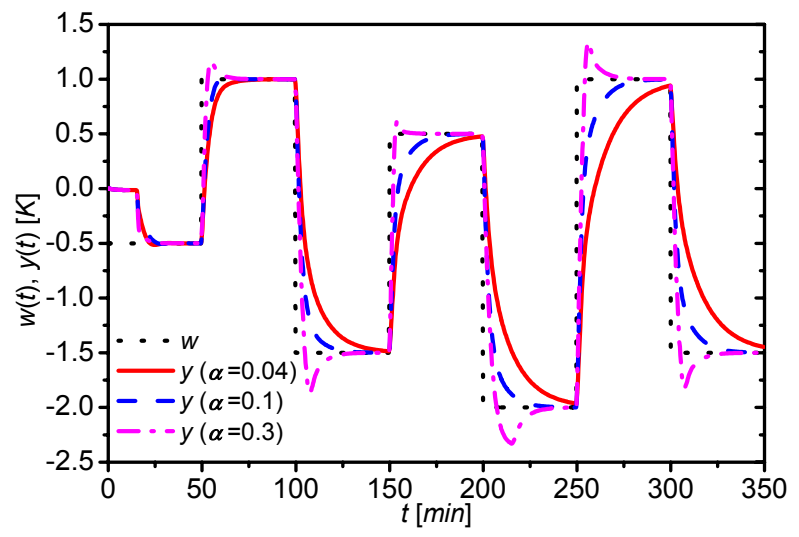

Figure 6: The course of the output variable, $y(t)$, for different value of the parameter $\alpha, 1^{\text {st }}$ order ELM $G_{1}$

The course of the identified parameters is shown in Figure 7.

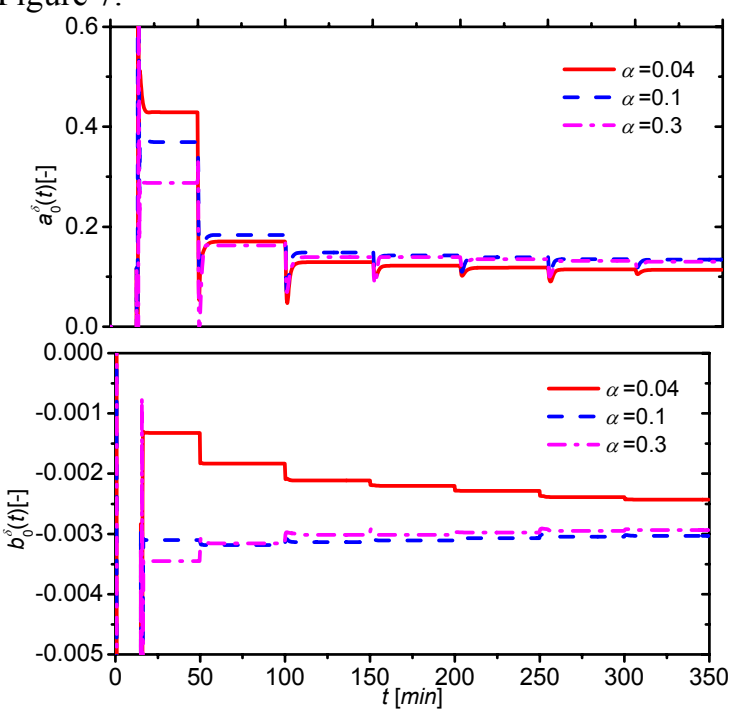

Figure 7: The course of the parameters $a_{0}{ }^{\delta}(t)$ a $b_{0}{ }^{\delta}(t)$ for different value of the parameter $\alpha, 1^{\text {st }}$ order ELM $G_{1}$

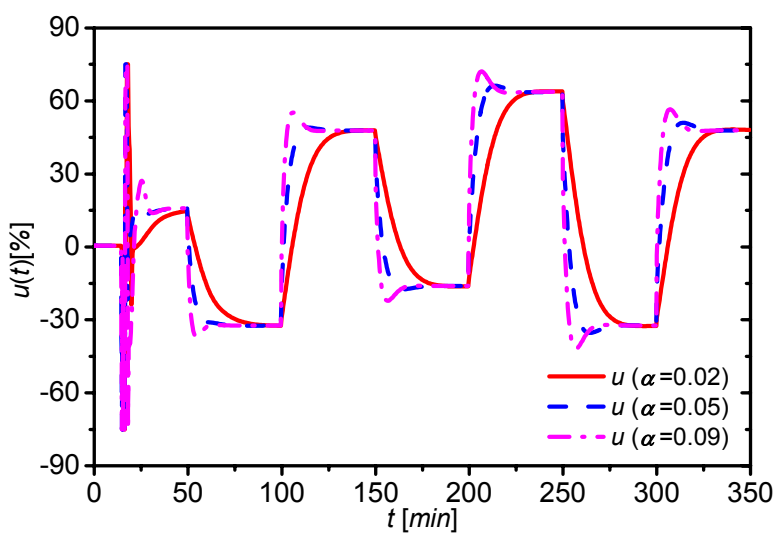

Figure 8: The course of the input variable, $u(t)$, for different value of the parameter $\alpha, 2^{\text {nd }}$ order ELM $G_{2}$
The second analysis was done for ELM $G_{2}(s)$ which is the second order transfer function with relative order one. Simulation studies for the same value of $\alpha$ as in previous study produces much slower output responses. Parameters $\alpha$ have not purely equal meaning in both studies because the first order transfer ELM makes the second degree polynomial $d(s)$ with single root $\alpha$ on the right side of the Diophantine equation (16) while the second order ELM means $d(s)$ of the fourth degree and $\alpha$ is double root. That is why this controller was tested for tuning parameter $\alpha=0.02,0.05$ and 0.09 which produces similar output responses as in the first case.

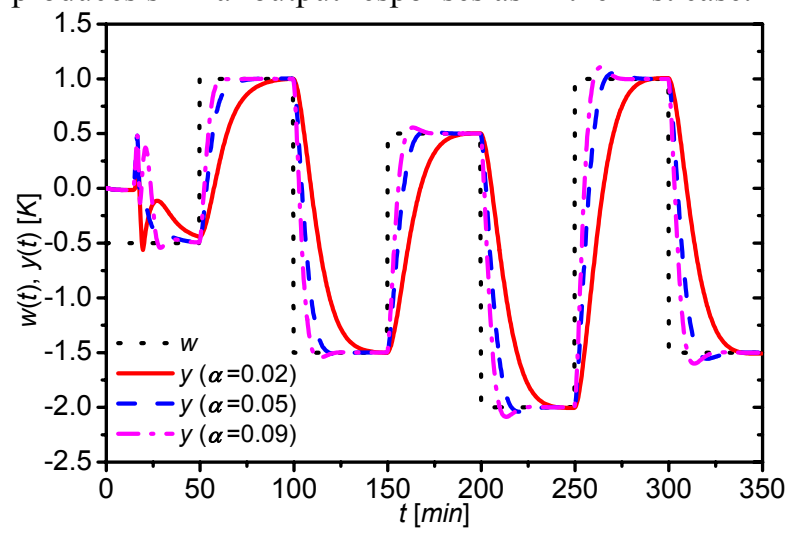

Figure 9: The course of the output variable, $y(t)$, for different value of the parameter $\alpha, 2^{\text {nd }}$ order ELM $G_{2}$

This controller have not very optimal results at the begging which was caused mainly by the recursive identification which again started from the general values $\quad \hat{\boldsymbol{\theta}}^{T}=[0.1,0.1,0.1,0.1]$ and employes the proportional controller at the beginning again. Even though the course of the input variable $u(t)$ in Figure 8 is very special and not very good, the resulting output response $y(t)$ is not as bad as we expect - see Figure 9.

Courses of the identified parameters during the control are confront in Figure 10 and Figure 11.

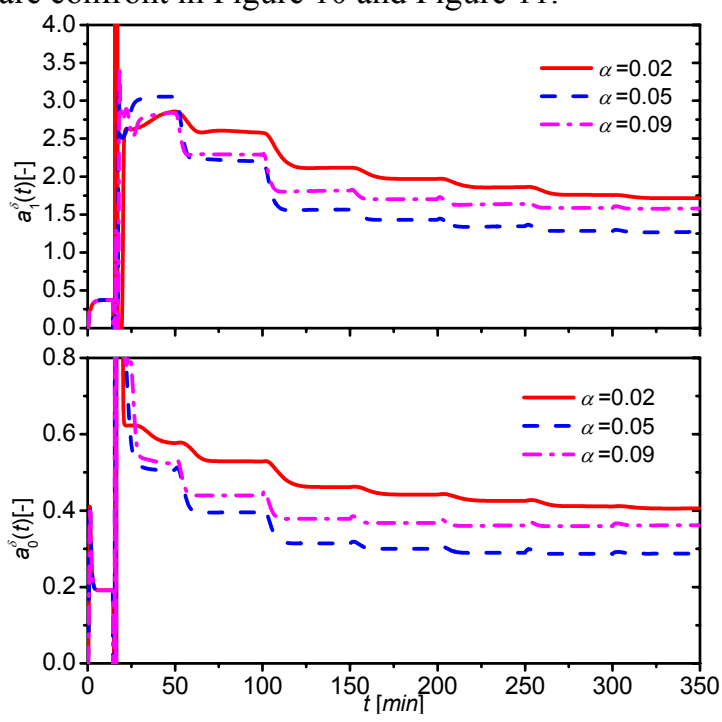

Figure 10: The course of the parameters $a_{1}{ }^{\delta}(t)$ a $a_{0}{ }^{\delta}(t)$ for different value of the parameter $\alpha, 2^{\text {nd }}$ order ELM $G_{2}$ 


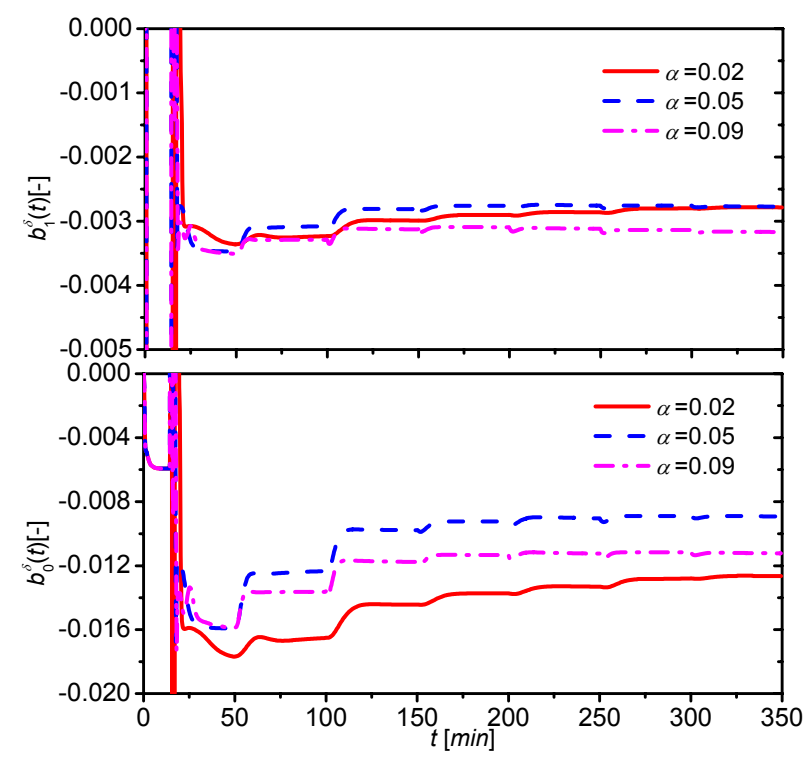

Figure 11: The course of the parameters $b_{1}{ }^{\delta}(t)$ a $b_{0}{ }^{\delta}(t)$ for different value of the parameter $\alpha, 2^{\text {nd }}$ order ELM $G_{2}$

It is good to qualify the results somehow. The quality criteria $S_{u}$ and $S_{y}$ were used in our case. These criteria are computed from

$$
\begin{aligned}
& S_{u}=\sum_{k_{1}+1}^{k_{2}}(u(i)-u(i-1))^{2}[-] \\
& S_{y}=\sum_{k_{1}}^{k_{2}}(w(i)-y(i))^{2}\left[K^{2}\right]
\end{aligned}
$$

where the computation interval $<k_{1} ; k_{2}>$ is $<50 ; 350>$ min due to unappropriate results at the beginning during the first step change. The resulting values of the criteria are in Table 3.

We can say that the compromise between the speed of the control and the control quality are the middle values of parameter $\alpha$, i.e. $\alpha=0.1$ for $1^{\text {st }}$ order ELM and $\alpha=0.05$ for $2^{\text {nd }}$ order ELM. These simulation results are compared in the last Figure 12 and Figure 13.

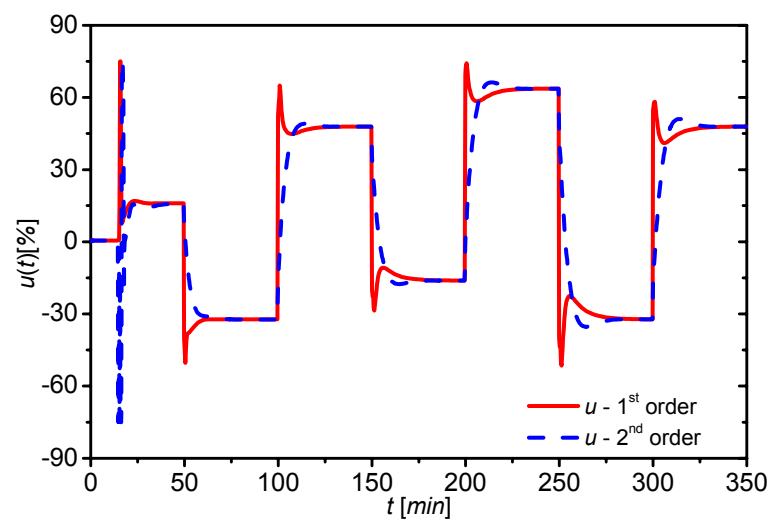

Figure 12: Comparison of the course of the input variable $u(t)$ for $1^{\text {st }}$ and $2^{\text {nd }}$ order ELM
Table 3: Control quality criteria $S_{u}$ and $S_{y}$

\begin{tabular}{|c|c|c|}
\cline { 2 - 3 } \multicolumn{1}{c|}{} & $S_{u}[-]$ & $S_{y}\left[K^{2}\right]$ \\
\hline \multicolumn{2}{c|}{ The first order transfer function $G_{1}(s)$} \\
\hline$\alpha=0.04$ & 15686 & 435.62 \\
$\alpha=0.1$ & 41171 & 218.96 \\
$\alpha=0.3$ & 75510 & 161.17 \\
\hline \multicolumn{2}{|c|}{ The second order transfer function $G_{2}(s)$} \\
\hline$\alpha=0.02$ & 518 & 961.72 \\
$\alpha=0.05$ & 2282 & 466.35 \\
$\alpha=0.09$ & 6178 & 312.07 \\
\hline
\end{tabular}

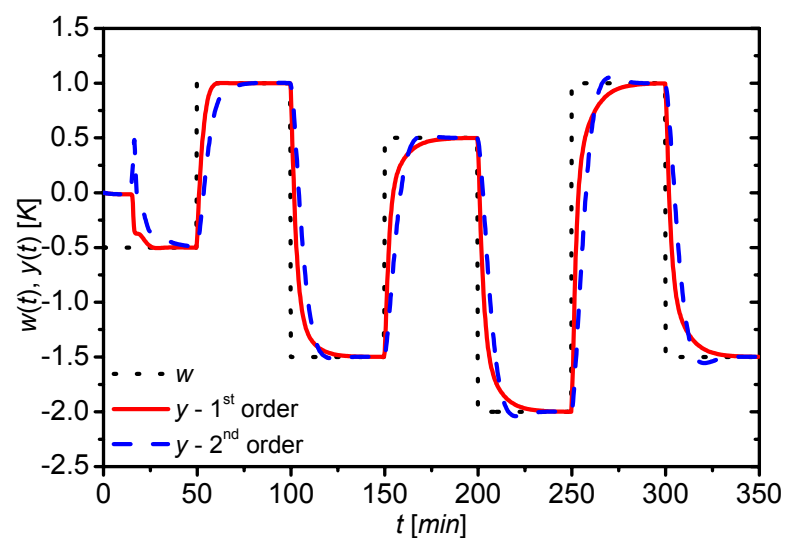

Figure 13: Comparison of the course of the output variable $y(t)$ for $1^{\text {st }}$ and $2^{\text {nd }}$ order ELM

\section{CONCLUSION}

This paper presents simulation results of the adaptive control of the nonlinear system based on the choice of the External Linear Model parameters of which are estimated during the control and parameters of the controller are then adopt to these identified ones. There were used two ELM differing in the order of the transfer function. Obtained results have shown that the more simple first order transfer function have very good control results and the estimation has no problem although it starts from the general values. The controller derived from the second order transfer function with relative order one as an ELM has a bit worthier results especialy at the very beginning of the control because of the unappropriate identification but the course of the input and the output variables after the second step change in the time $t=50 \mathrm{~min}$ is then similarly good as in previous case. The speed and the quality of the control could be affected by the choice of the parameter $\alpha$ while decreasing value results in steepening output response but overshoots. Although this system has nonlinear behavior and other negative control properties, both proposing controllers produces good control results. The first order ELM could be in this case better choice from the computing, programming and practical point of view. 


\section{REFERENCES}

Åström, K.J. and B. Wittenmark 1989. Adaptive Control. Addison Wesley. Reading. MA.

Bobal, V.; J. Böhm; J. Fessl; and J. Machacek 2005. Digital Self-tuning Controllers: Algorithms. Implementation and Applications. Advanced Textbooks in Control and Signal Processing. Springer-Verlag London Limited

Ingham, J.; I. J. Dunn; E. Heinzle; and J. E. Přenosil 2000. Chemical Engineering Dynamics. An Introduction to Modeling and Computer Simulation. Second. Completely Revised Edition. VCH Verlagsgesellshaft. Weinheim.

Kucera, V. 1993. Diophantine equations in control - A survey. Automatica. 29. 1361-1375

Middleton, R.H. and G. C. Goodwin 2004 Digital Control and Estimation - A Unified Approach. Prentice Hall. Englewood Cliffs.

Mukhopadhyay, S.; A. G. Patra; and G. P. Rao 1992. New class of discrete-time models for continuos-time systeme. International Journal of Control. vol.55. 1161-1187

Rao, G.P. and H. Unbehauen, H. 2005. Identification of continuous-time systems. IEE Proc.-Control Theory Appl., 152, 185-220

Stericker, D.L. and N. K. Sinha 1993. Identification of continuous-time systems from samples of input-output data using the $\delta$-operator. Control-Theory and Advanced Technology. vol. 9. 113-125
Vojtesek, J.; P. Dostal; and M. Navratil 2008. Adaptive Control of CSTR with Van der Vusse Reaction. In: Proceedings 35th International Coference of Slovak Society of Chemical Engineering, Tatranske Matliare, Slovakia

\section{AUTHOR BIOGRAPHIES}

JIRI VOJTESEK was born in Zlin. Czech Republic and studied at the Tomas Bata University in Z Zlin. where he got his master degree in chemical and process engineering in 2002. $\mathrm{He}$ has finished his Ph.D. focused on Modern control methods for chemical reactors in 2007. His contact is vojtesek@fai.utb.cz.

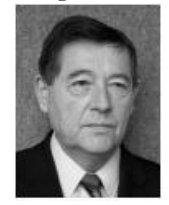

PETR DOSTAL studied at the Technical University of Pardubice. He obtained his PhD. degree in Technical Cybernetics in 1979 and he became professor in Process Control in 2000. His research interest are modeling and simulation of continuous-time chemical processes. polynomial methods. optimal. adaptive and robust control. You can contact him on email address dostalp@fai.utb.cz. 